\title{
Alleviation of Salinity Stress During Seed Germination and Early Growth Stage in Sweet Pepper by Seed Priming with Acetyl Salicylic Acid El-Afifi, S.T. ${ }^{1}$; M. M. Zaghloul ${ }^{1}$; H. M. Ahmed ${ }^{2}$; and M. A. Mohamed ${ }^{2}$ \\ ${ }^{1}$ Dept. of Vegetable and Floriculture, Faculty of Agriculture, Mansoura University, Egypt. \\ ${ }^{2}$ Dept. of Vegetable Crops Seed Production and Technology, HRI, Agricultural Research Center, Giza, Egypt.
}

\begin{abstract}
To investigate the interaction between salinity stress and seed priming with acetyl salicylic acid on sweet pepper seed germination and early growth, two factorial experiments were carried out during the two seasons 2014 and 2015 . A lab experiment involved seed priming in four different concentrations $0.0,0.1,0.3$ and $0.5 \mathrm{mM}$ of acetyl salicylic acid, the performance of primed seeds was assessed under 4 different salinity levels $0,30,60$, and $90 \mathrm{mM} \mathrm{NaCl}$. The second experiment was carried out in foam transplanting trays under greenhouse conditions. Primed seeds were irrigated with $0,30,60$, and $90 \mathrm{mM}$ $\mathrm{NaCl}$ solutions. The obtained results showed that salinity reduced germination percentage, delayed germination, and reduced seedling and transplant growth parameters. Nutrients uptake was reduced by salinity increasing. Different concentrations acetyl salicylic acid had relieving effect. Among all concentration $0.3 \mathrm{mM}$ acetyl salicylic acid was the best concentration in most cases. Keywords: salinity, abiotic, stress, vigor, transplant, pepper, emergence, germination
\end{abstract}

\section{INTRODUCTION}

Salinization is one of the major crop productivity limiting factors particularly in arid and semi-arid characterized with limited water resources (Munns and Tester, 2008). In Egypt, as most of arable lands are irrigated, $33 \%$ of cultivated lands are salt-effected (Mohamed et al., 2007). Sweet pepper is considered one of the most important vegetable crops in arid and semi-arid regions suffering from salinity problems, it is considered sensitive to salinity (Kurunc et al., 2011; Pinero et al., 2014). Although pepper genotypes vary in their tolerance to salinity(Aktas et al., 2006), salinity resistance threshold found to be $1.5 \mathrm{dS} \mathrm{m}^{-1}$, below which no negative effect on growth and a $14 \%$ reduction in biomass production for each additional 1 $\mathrm{dS} \mathrm{m}^{-1}$ were observed (Maas and Hoffman, 1977; Rhoades et al., 1992). Seed germination and early seedling growth are considered as the most sensitive two stages to salinity stress (Ashraf and Foolad, 2005). Also, seed germination and seedling emergence of pepper is slow and non-uniform under normal as well as adverse conditions (Demir and Okcu, 2004). Low $\mathrm{NaCl}$ concentrations did not affect pepper seed germination percentage, however with salinity level increasing; the ability of seeds to germinate was significantly reduced. Negative effect was more pronounced on radicale length and seedling leaf area (Chartzoulakis and Klapaki, 2000). Among Various techniques employed to improve seed germination, emergence and stand establishment under salt conditions, seed priming is one of the most effective and frequently utilized techniques. It enhances the speed and uniformity of germination, that results in faster and better germination in different crops (Cantliffe, 2003). It helps in seedling development in a wide range of conditions and decreases sensitivity to external factors (Ashraf and Foolad, 2005; Ibrahim, 2016). Inclusion of plant growth regulators and hormones during priming and other pre-sowing treatments can be improve Seed performance of different crops (Lee et al., 1998). Hormone like salicylic acid and its derivative acetyl salicylic acid have also proved alleviating cold stress on germination and emergence of sweet pepper (Korkmaz, 2005), acetyl salicylic acid has protected muskmelon seedlings against drought stress (Korkmaz et al., 2007). Also, salicylic acid and acetyl salicylic acid have been showed to decrease the harmful effects of abiotic stress on tomato and bean plants (Senaratna et al., 2000). In hot pepper, seed priming with both salicylic acid and acetyl salicylic acid could improve uniformity and seedling establishment under nonsaline and saline conditions (Khan et al., 2009). Exogenous SA improved wheat seed germination, seedling growth, fresh weight, and dry weight of seedlings; enhanced cell division and extension of root; improved yield; and ameliorated drought and salt stress (Shakirova and Sakhabutdinova, 2003). Therefore, the purpose of the current study is to study the response of sweet pepper during seed germination and early growth stages to various salinity levels, Moreover, to figure out the most appropriate concentration of acetyl salicylic acid, as a priming agents, to alleviate salinity adverse effects on sweet pepper.

\section{MATERIALS AND METHODS}

In this study, two experiments were carried out. The first was a laboratory experiment. It was carried out in the lab of seed technology, Department of Vegetable Crops Seed Science and Technology, Horticulture Research Institute, Agriculture Research Center, Giza, Egypt to study the germination characteristics. The second was a seedling trays experiment done under greenhouse conditions at a private nursery, Baramon, Mansoura, Egypt to study emergence behavior and transplant characteristics.

Seeds of pepper cv Orlando were surface sterilized by dipping in sodium hypochlorite $(5 \%)$ solution for 5 minutes, washed three times with tap water then rinsed with distilled water, and dried by blotting in filter paper. These surface sterilized seeds 
were divided to four portions. Three portions were primed in aerated solution of acetyl salicylic acid at concentration of $0.1,0.3,0.5 \mathrm{mM}$, while the fourth portion was primed in distilled water $(0.0 \mathrm{mM}$ ASA) to serve as control. Seeds were primed for $36 \mathrm{~h}$ at $25 \pm$ $2^{\circ} \mathrm{C}$ under dark conditions. After priming, seeds were washed with distilled water. Then the seeds were dried at room temperature between filter paper, after that packed in polythene bags and kept in a refrigerator at 5 ${ }^{\circ} \mathrm{C}$ for further use.

laboratory experiment was repeated twice, during February 2014 and February 2015: Primed seeds were sown in $90 \mathrm{~mm}$ diameter Petri dishes top of two layers of Whatman No. 1 filter paper, moistened with $5 \mathrm{ml}$ of one of $0.0,30.0,60.0$ and $90.0 \mathrm{mM} \mathrm{NaCl}$ solutions respectively, at $25 \pm 2^{\circ} \mathrm{C}$ and kept under 16 hours photoperiod, treatments were replicated 4 times, 50 seeds per each. Data on germination were recorded daily for 14 days; seed with $2 \mathrm{~mm}$ radicle protrusion was considered as germinated. Seven days old seedlings were used to measure radicle and plumule lengths, fresh and dry weights of seedlings.

Germination percentage was computed following the (ISTA, 2011)

$$
\mathbf{G P}=\frac{\text { Germinated Seeds No. }}{\text { Total Number of Seeds }} \times 100
$$

Mean germination time (MGT) was calculated according to the equation of Ellis and Roberts (1981):

$$
\text { MGT }=\sum D \mathbf{D} / \sum \mathbf{n}
$$

Where ' $n$ ' is the number of seeds germinated on day $\mathrm{D}$, where $\mathrm{D}$ is number of days counted from the beginning of germination.

Germination performance index (GPI) was calculated according to formula: GPI= GP/MGT

Where GP is final germination percentage and MGT is mean germination time in days, Pill and Fieldhouse (1982).

The time taken to $50 \%$ germination (T50) was calculated according to the formula modified by (Farooq et al., 2005)

$$
\mathbf{T}_{50}=\mathbf{t}_{\mathrm{i}}+\left[(\mathbf{N} / 2)-\boldsymbol{n}_{i}\right]\left(\boldsymbol{t}_{j}-\boldsymbol{t}_{i}\right) / \mathbf{n}_{\mathrm{j}}-\mathbf{n}_{\mathrm{i}}
$$

Where $\mathrm{N}$ is the final number of germinated seeds and $\mathrm{n}_{\mathrm{i}}$ and $\mathrm{n}_{\mathrm{j}}$ are the cumulative number of seeds germinated by adjacent seed count at times $t_{i}$ and $t_{j}$ respectively, when $n_{i}<N / 2<n_{j}$.

Coefficient of Velocity (CoV) was calculated according to the formula: $\mathrm{CoV}=1 / \mathrm{MGT}$ X 100

Where MGT is mean germination time in days (Edwards and Sundstorm, 1987).

Vigor index was calculated in two ways by the following formulae:

Vigor Index 1= Final Germination $(\%) \times$ Total Seedling Length (cm)

Vigor Index 2 = Final Germination $(\%) \times$ Seedling dry weight (mg)

(Abdul-Baki and Anderson, 1973)

The transplant experiment was repeated twice through March and April 2014 and 2015, primed seed were sown in 209 cell foam trays filled with fertilized potting media constitutes of 1: 1 peat moss/ vermiculite $(\mathrm{v}: \mathrm{v})$, irrigated with the abovementioned salt water and incubated until seedling emergence initiated. The final emergence percentage was calculated, it was used in correlation and regression purpose. Transplant Height $(\mathrm{cm})$ and transplant dry weight $(\mathrm{g})$ were evaluated on 40 days old transplants. Chemical composition of leaves were analyzed; proline content was estimated following (Bates et al., 1973) . Nitrogen and phosphorus were calorimetrically determined according the methods described in (A.O.A.C., 1992). Potassium was measured using the flame photometer according to (Chapman and Pratt, 1961). Data were expressed as \% of dry matter.

The experimental design followed was factorial in completely randomized design with four replicates. The recorded data were statistically analyzed using general linear model of Statistix 8.0 and treatments means were separated using DMR test $(\mathrm{p}<0.05)$.

\section{RESULTS AND DISCUSSION}

Sweet pepper is considered either sensitive or moderately sensitive to salinity (Cornillon and Palloix, 1995 and Rhoades et al.,, 1992). During plant life, Seed germination and seedling growth are of the stages most sensitive to salinity. It delays or prevents the seed germination through various aspects, such as a reduction in water availability, ion toxicity, induction of oxidative stress, distribution in the mobilization of stored reserves and affecting the structural organization of proteins (Ibrahim, 2016). Data of laboratory experiment shown in table 1 reveal no significant difference between non-saline and low salinity on germination percentage in both seasons. With the increase of salinity level, a significant reduction was observed in GP. This maximized when seeds were germinated under $90 \mathrm{mM} \mathrm{NaCl}$ hereafter called high salinity conditions, as germinated seeds were reduced from $80.32 \%$ under non-stress conditions to $58.16 \%$ under the high salinity stress. These findings are in agree with those of (Chartzoulakis and Klapaki, 2000; Yildirim and Güvenç, 2006). Regardless salinity level, seed priming in various concentrations of acetyl salicylic acid (ASA) offered a potential role in alleviating the negative effect of salinity on germination percentage , all treatments surpassed $0.0 \mathrm{mM}$ ASA (control) and $0.3 \mathrm{mM}$ ASA was the superior treatment recording an increase by 26.13 and $26.14 \%$ over control in the first and second season respectively, similar to (Khan et al., 2009). The interaction between two factors varied due to the salinity level and priming treatment, it was in favor of $30 \mathrm{mM} \mathrm{NaCl}$ and $0.3 \mathrm{mM}$ ASA as GP reached $88.88 \%$ and the least GP was observed when water primed seeds were germinated under high salinity to reach $47.81 \%$. There was no significant difference between water primed seeds germinated under $60 \mathrm{mM}$ $\mathrm{NaCl}$, hereafter, moderate salinity and $0.3 \mathrm{mM}$ ASA primed seeds under high salinity. This is remarkable, primed seed can be germinated under high salinity without significant loss in GP. and these agree with results of (Gain et al., 2004; Iroka et al., 2016). 
Table 1. Effect of salinity, acetyl salicylic acid seed priming and their interaction on germination parameters during two seasons 2014 and 2015.

\begin{tabular}{|c|c|c|c|c|c|c|c|c|c|c|c|}
\hline \multicolumn{2}{|c|}{ Treatments } & \multicolumn{2}{|c|}{$\begin{array}{c}\text { Germination } \\
\text { percentage }(\%)\end{array}$} & \multicolumn{2}{|c|}{$\begin{array}{l}\text { MGT } \\
\text { (days) }\end{array}$} & \multicolumn{2}{|c|}{ GPI } & \multicolumn{2}{|c|}{$\begin{array}{c}\text { T50 } \\
\text { (days) }\end{array}$} & \multicolumn{2}{|c|}{$\begin{array}{l}\text { Coefficient of } \\
\text { velocity }\end{array}$} \\
\hline $\begin{array}{l}\text { Salinity } \\
\text { level }\end{array}$ & $\begin{array}{c}\text { Seed } \\
\text { priming }\end{array}$ & $\begin{array}{c}\text { 1st } \\
\text { season }\end{array}$ & $\begin{array}{c}\text { 2nd } \\
\text { season }\end{array}$ & $\begin{array}{c}1^{\text {st }} \\
\text { season }\end{array}$ & $\begin{array}{c}\text { 2nd } \\
\text { season }\end{array}$ & $\begin{array}{c}1 \text { st } \\
\text { season }\end{array}$ & $\begin{array}{l}\text { 2nd } \\
\text { season }\end{array}$ & $\begin{array}{c}\text { 1st } \\
\text { season }\end{array}$ & $\begin{array}{c}\text { 2nd } \\
\text { season }\end{array}$ & $\begin{array}{c}\text { 1st } \\
\text { season }\end{array}$ & $\begin{array}{c}2^{\text {nd }} \\
\text { season }\end{array}$ \\
\hline \multicolumn{12}{|c|}{ Salinity level $(\mathrm{mM} \mathrm{NaCl})$} \\
\hline $0 \mathrm{mM}$ & & $80.32 \mathrm{a}$ & $78.71 \mathrm{a}$ & $5.00 \mathrm{~d}$ & $4.75 \mathrm{~d}$ & $17.26 \mathrm{a}$ & & $4.97 \mathrm{~d}$ & $4.77 \mathrm{~d}$ & $21.07 \mathrm{a}$ & $22.18 \mathrm{a}$ \\
\hline $30 \mathrm{mM}$ & & $81.74 \mathrm{a}$ & 80.1 & $5.81 \mathrm{c}$ & $52 \mathrm{c}$ & $15.48 \mathrm{~b}$ & & $5.15 \mathrm{c}$ & $4.94 \mathrm{c}$ & $18.65 \mathrm{~b}$ & $19.63 \mathrm{~b}$ \\
\hline $60 \mathrm{mM}$ & & $73.34 \mathrm{~b}$ & 71.8 & $7.05 \mathrm{~b}$ & & $11.15 \mathrm{c}$ & & $5.81 \mathrm{~b}$ & $5.57 \mathrm{~b}$ & $14.99 \mathrm{c}$ & $15.78 \mathrm{c}$ \\
\hline $0 \mathrm{mM}$ & & $58.16 \mathrm{c}$ & $57.00 \mathrm{c}$ & $9.08 \mathrm{a}$ & $8.63 \mathrm{a}$ & $6.90 \mathrm{~d}$ & $7.11 \mathrm{~d}$ & $6.61 \mathrm{a}$ & $6.34 \mathrm{a}$ & $11.60 \mathrm{~d}$ & $12.21 \mathrm{~d}$ \\
\hline \multicolumn{12}{|c|}{ Seed priming (mM Acetyl Salicylic Acid) } \\
\hline & $0.0 \mathrm{mM}$ & $63.73 \mathrm{c}$ & $62.45 \mathrm{c}$ & $9.77 \mathrm{a}$ & $9.28 \mathrm{a}$ & $7.06 \mathrm{c}$ & $7.28 \mathrm{c}$ & $8.73 \mathrm{a}$ & $8.38 \mathrm{a}$ & $10.77 \mathrm{c}$ & $11.33 \mathrm{c}$ \\
\hline & $0.1 \mathrm{mM}$ & $75.29 \mathrm{~b}$ & 73.7 & $6.05 \mathrm{~b}$ & & $13.49 \mathrm{~b}$ & & $5.71 \mathrm{~b}$ & $5.48 \mathrm{~b}$ & $17.50 \mathrm{~b}$ & $18.42 \mathrm{~b}$ \\
\hline & $0.3 \mathrm{mM}$ & $80.38 \mathrm{a}$ & 78. & $23 \mathrm{~d}$ & & 94 a & & $3.56 \mathrm{~d}$ & $3.42 \mathrm{c}$ & $20.42 \mathrm{a}$ & $21.50 \mathrm{a}$ \\
\hline & & $74.15 \mathrm{~b}$ & & $5.90 \mathrm{c}$ & $5.60 \mathrm{c}$ & $13.30 \mathrm{~b}$ & & $4.53 \mathrm{c}$ & $4.35 \mathrm{~d}$ & $17.62 \mathrm{~b}$ & $18.55 \mathrm{~b}$ \\
\hline \multicolumn{12}{|c|}{ Interaction (salinity * seed priming) } \\
\hline \multirow{4}{*}{$0 \mathrm{mM}$} & $0.0 \mathrm{mM}$ & $70.33 \mathrm{f}$ & & $6.99 \mathrm{~g}$ & $6.64 \mathrm{~g}$ & $10.07 \mathrm{~g}$ & $10.39 \mathrm{~g}$ & $6.99 \mathrm{c}$ & $6.71 \mathrm{c}$ & $14.32 \mathrm{fg}$ & $15.07 \mathrm{fg}$ \\
\hline & $0.1 \mathrm{mM}$ & $80.27 \mathrm{bc}$ & $78.66 \mathrm{bc}$ & 4.651 & 4.421 & $17.27 \mathrm{c}$ & $17.81 \mathrm{c}$ & $5.62 \mathrm{e}$ & $5.39 \mathrm{e}$ & $21.50 \mathrm{c}$ & $22.63 \mathrm{c}$ \\
\hline & $0.3 \mathrm{mM}$ & $92.24 \mathrm{a}$ & $90.40 \mathrm{a}$ & $3.75 \mathrm{n}$ & $3.56 \mathrm{n}$ & $24.62 \mathrm{a}$ & $25.40 \mathrm{a}$ & $3.10 \mathrm{j}$ & $2.97 \mathrm{i}$ & $26.70 \mathrm{a}$ & $28.10 \mathrm{a}$ \\
\hline & $0.5 \mathrm{mM}$ & $78.42 \mathrm{~cd}$ & $76.85 \mathrm{~cd}$ & $4.59 \mathrm{~lm}$ & $4.36 \mathrm{~lm}$ & $17.08 \mathrm{~cd}$ & $17.62 \mathrm{~cd}$ & $4.17 \mathrm{~h}$ & $4.00 \mathrm{~h}$ & $21.78 \mathrm{c}$ & $22.92 \mathrm{c}$ \\
\hline \multirow{4}{*}{$30 \mathrm{mM}$} & $0.0 \mathrm{mM}$ & 73.75 ef & 72.27 ef & $8.93 \mathrm{c}$ & $8.49 \mathrm{c}$ & $8.25 \mathrm{~h}$ & $8.52 \mathrm{~h}$ & $7.19 \mathrm{c}$ & $6.91 \mathrm{c}$ & $11.19 \mathrm{j}$ & $11.79 \mathrm{j}$ \\
\hline & $0.1 \mathrm{mM}$ & $81.52 \mathrm{bc}$ & $79.88 \mathrm{bc}$ & 4.751 & 4.511 & $17.17 \mathrm{c}$ & $17.71 \mathrm{c}$ & $5.85 \mathrm{e}$ & $5.61 \mathrm{e}$ & $21.06 \mathrm{c}$ & $22.17 \mathrm{c}$ \\
\hline & $0.3 \mathrm{mM}$ & $88.88 \mathrm{a}$ & $87.10 \mathrm{a}$ & $4.36 \mathrm{~m}$ & $4.15 \mathrm{~m}$ & $20.53 \mathrm{~b}$ & $21.18 \mathrm{~b}$ & $3.34 \mathrm{i}$ & $3.21 \mathrm{i}$ & $23.09 \mathrm{~b}$ & $24.31 \mathrm{~b}$ \\
\hline & $0.5 \mathrm{mM}$ & $82.83 \mathrm{~b}$ & $81.17 \mathrm{~b}$ & $5.19 \mathrm{k}$ & $4.93 \mathrm{k}$ & $15.96 \mathrm{~d}$ & $16.46 \mathrm{~d}$ & $4.22 \mathrm{gh}$ & $4.05 \mathrm{gh}$ & $19.26 \mathrm{~d}$ & $20.27 \mathrm{~d}$ \\
\hline \multirow{4}{*}{$60 \mathrm{mM}$} & $0.0 \mathrm{mM}$ & $63.03 \mathrm{~g}$ & $61.77 \mathrm{~g}$ & $10.13 \mathrm{~b}$ & $9.63 \mathrm{~b}$ & $6.22 \mathrm{j}$ & $6.42 \mathrm{j}$ & $9.14 \mathrm{~b}$ & $8.77 \mathrm{~b}$ & $9.87 \mathrm{k}$ & $10.39 \mathrm{k}$ \\
\hline & $0.1 \mathrm{mM}$ & $81.82 \mathrm{bc}$ & $80.18 \mathrm{bc}$ & $6.53 \mathrm{~h}$ & $6.21 \mathrm{~h}$ & $12.54 \mathrm{ef}$ & 12.93 ef & $6.22 \mathrm{~d}$ & $5.97 \mathrm{~d}$ & $15.31 \mathrm{f}$ & $16.12 \mathrm{f}$ \\
\hline & $0.3 \mathrm{mM}$ & $74.75 \mathrm{de}$ & $73.25 \mathrm{de}$ & & & & & $3.44 \mathrm{i}$ & $3.30 \mathrm{i}$ & $18.18 \mathrm{~d}$ & $19.14 \mathrm{~d}$ \\
\hline & $0.5 \mathrm{mM}$ & 73.74 ef & $72.26 \mathrm{ef}$ & $6.03 \mathrm{i}$ & $5.73 \mathrm{i}$ & $12.24 \mathrm{f}$ & $12.63 \mathrm{f}$ & $4.43 \mathrm{~g}$ & $4.25 \mathrm{~g}$ & $16.60 \mathrm{e}$ & $17.47 \mathrm{e}$ \\
\hline \multirow{4}{*}{$90 \mathrm{mM}$} & $0.0 \mathrm{mM}$ & $47.81 \mathrm{i}$ & $46.85 \mathrm{I} \mathrm{i}$ & $13.01 \mathrm{a}$ & $12.36 \mathrm{a}$ & $3.68 \mathrm{k}$ & $3.79 \mathrm{k}$ & $11.62 \mathrm{a}$ & $11.15 \mathrm{a}$ & 7.691 & 8.091 \\
\hline & $0.1 \mathrm{mM}$ & $57.57 \mathrm{~h}$ & $56.41 \mathrm{~h}$ & $8.24 \mathrm{~d}$ & $7.83 \mathrm{~d}$ & $6.99 \mathrm{ij}$ & $7.21 \mathrm{ij}$ & $5.14 \mathrm{f}$ & $4.93 \mathrm{f}$ & $12.13 \mathrm{ij}$ & $12.77 \mathrm{ij}$ \\
\hline & $0.3 \mathrm{mM}$ & $65.66 \mathrm{~g}$ & $64.34 \mathrm{~g}$ & $7.30 \mathrm{f}$ & $6.94 \mathrm{f}$ & $9.00 \mathrm{gh}$ & $9.28 \mathrm{gh}$ & $4.37 \mathrm{gh}$ & $4.20 \mathrm{gh}$ & $13.71 \mathrm{gh}$ & $14.43 \mathrm{gh}$ \\
\hline & $0.5 \mathrm{mM}$ & $61.62 \mathrm{gh}$ & $60.38 \mathrm{gh}$ & $7.78 \mathrm{e}$ & $7.39 \mathrm{e}$ & $7.92 \mathrm{hi}$ & $8.17 \mathrm{hi}$ & $5.29 \mathrm{f}$ & $5.08 \mathrm{f}$ & $12.86 \mathrm{hi}$ & $13.54 \mathrm{hi}$ \\
\hline
\end{tabular}

MGT: mean germination time, GPI: germination performance index, $\mathrm{T}_{50}$ : time required for $50 \%$ of germination

Mean germination time (MGT) and $\mathrm{T}_{50}$ as temporal parameters responded positively to salinity, the higher the salinity the longer the time required, in both seasons. This means delayed germination and these findings agree with (Khan et al., 2009). Regardless, salinity level, ASA could diminish both of MGT and $\mathrm{T}_{50}$ to $54 \%$ and $41 \%$ respectively, of the time required for control treatments which means faster germination. The interaction between two factors revealed that the most favorable results obtained when $0.3 \mathrm{mM}$ ASA primed seeds were germinated under control conditions. Furthermore, priming seeds in $0.3 \mathrm{mM}$ ASA could minimize the $\mathrm{T}_{50}$ regardless salinity level, which means that ASA seed priming is a potential technique to speed up germination process these findings agree with (Khan $e t$ al.,, 2009). Germination performance index GPI and coefficient of velocity $\mathrm{CoV}$ as two characteristics of germination quality had the same trend, they were reduced with the increase in salinity level. The lowest values recorded in both parameters were gotten under high salinity in the two seasons. Similarly they were responded positively to ASA priming treatments (Cantliffe, 2003).
Table 2 shows the results of seedling parameters and vigor index values estimated in 2 ways. All estimated values insure the negative impact of salinity on the abovementioned parameters. For instance, mean seedling length decreased from 9.59 to 8.66 and from 9.23 to $8.34 \mathrm{~cm}$ under control and high salinity in two seasons respectively and similarly were values of seedling fresh and dry weight, and vigor index 1 and 2. Regardless salinity effect, seed priming in ASA recorded improvements over control in all parameters and the highest values were in favor 0.3 mM ASA priming treatment these results agree with those published by (Chartzoulakis and Klapaki, 2000; Khan et al., 2009). The interaction between seed priming and salinity revealed lowest values were recorded in the two seasons when water primed seeds were germinated under high salinity. Moreover, all of priming treatments improved seedling and vigor indices over control; this confirms that ASA seed priming can be potentially employed in enhancement of seedling characteristics and vigor indices under salinity stress conditions. 
El-Afifi, S.T. et al.

Table 2. Effect of salinity, acetyl salicylic acid seed priming and their interaction on seedling characteristics and vigor indices during two seasons 2014 and 2015.

\begin{tabular}{|c|c|c|c|c|c|c|c|c|c|c|c|}
\hline \multicolumn{2}{|c|}{ Treatments } & \multicolumn{2}{|c|}{ Seedling length $(\mathrm{cm})$} & \multicolumn{2}{|c|}{ Seedling FW(mg) } & \multicolumn{2}{|c|}{ Seedling DW(mg) } & \multicolumn{2}{|c|}{ Vigor Index 1} & \multicolumn{2}{|c|}{ Vigor Index2 } \\
\hline $\begin{array}{l}\text { Salinity } \\
\text { level }\end{array}$ & $\begin{array}{c}\text { Seed } \\
\text { priming }\end{array}$ & $\begin{array}{c}\text { 1st } \\
\text { season }\end{array}$ & $\begin{array}{c}2^{\text {nd }} \\
\text { season }\end{array}$ & $\begin{array}{c}1 \text { 1st } \\
\text { season }\end{array}$ & $\begin{array}{c}\text { 2nd } \\
\text { season }\end{array}$ & $\begin{array}{c}\text { 1st } \\
\text { season }\end{array}$ & $\begin{array}{c}\text { 2nd } \\
\text { season }\end{array}$ & $\begin{array}{c}\text { 1st } \\
\text { season }\end{array}$ & $\begin{array}{c}\text { 2nd } \\
\text { season }\end{array}$ & $\begin{array}{c}1 \text { 1st } \\
\text { season }\end{array}$ & $\begin{array}{c}\text { 2nd } \\
\text { season }\end{array}$ \\
\hline \multicolumn{12}{|c|}{ Salinity level $(\mathrm{mM} \mathrm{NaCl})$} \\
\hline $0 \mathrm{mM}$ & & $9.59 \mathrm{a}$ & $9.23 \mathrm{a}$ & $33.824 \mathrm{~b}$ & $32.471 b$ & $2.411 \mathrm{~b}$ & $2.267 \mathrm{~b}$ & $729.00 \mathrm{a}$ & $772.86 \mathrm{a}$ & $178.93 \mathrm{~b}$ & $194.24 \mathrm{~b}$ \\
\hline $30 \mathrm{mM}$ & & $9.59 \mathrm{a}$ & $9.23 \mathrm{a}$ & $34.457 \mathrm{a}$ & $33.077 \mathrm{a}$ & $2.494 \mathrm{a}$ & $2.343 \mathrm{a}$ & $741.21 \mathrm{a}$ & $785.76 \mathrm{a}$ & $188.45 \mathrm{a}$ & $204.57 \mathrm{a}$ \\
\hline $60 \mathrm{mM}$ & & $9.10 \mathrm{~b}$ & $8.77 \mathrm{~b}$ & $33.614 \mathrm{c}$ & $32.270 \mathrm{c}$ & $2.537 \mathrm{a}$ & $2.385 \mathrm{a}$ & $630.93 \mathrm{~b}$ & $668.82 \mathrm{~b}$ & $171.83 \mathrm{c}$ & $186.53 \mathrm{c}$ \\
\hline $90 \mathrm{mM}$ & & $8.66 \mathrm{c}$ & $8.34 \mathrm{c}$ & $30.703 \mathrm{~d}$ & $29.474 \mathrm{~d}$ & $2.366 \mathrm{~b}$ & $2.223 \mathrm{~b}$ & $477.35 \mathrm{c}$ & $505.98 \mathrm{c}$ & $128.16 \mathrm{~d}$ & $139.13 \mathrm{~d}$ \\
\hline \multicolumn{12}{|c|}{ Seed priming (mM Acetyl Salicylic Acid) } \\
\hline & $0.0 \mathrm{mM}$ & $8.73 \mathrm{~d}$ & $8.40 \mathrm{~d}$ & $30.363 \mathrm{~d}$ & $29.148 \mathrm{~d}$ & $2.211 \mathrm{c}$ & $2.078 \mathrm{c}$ & $527.98 \mathrm{c}$ & $559.70 \mathrm{c}$ & $130.57 \mathrm{~d}$ & $141.73 \mathrm{~d}$ \\
\hline & & $9.16 \mathrm{c}$ & $82 \mathrm{c}$ & 32.57 & & & $78 \mathrm{~b}$ & $653.88 \mathrm{~b}$ & $693.16 \mathrm{~b}$ & $169.09 \mathrm{c}$ & $3.55 \mathrm{c}$ \\
\hline & $0.3 \mathrm{n}$ & $9.69 \mathrm{a}$ & 9.33 a & $35.898 \mathrm{a}$ & & 4 a & 38 a & $738.52 \mathrm{a}$ & $782.90 \mathrm{a}$ & $191.48 \mathrm{a}$ & $207.86 \mathrm{a}$ \\
\hline & $0.5 \mathrm{mM}$ & $9.37 \mathrm{~b}$ & $9.02 \mathrm{~b}$ & $33.764 \mathrm{~b}$ & $32.413 \mathrm{~b}$ & $2.578 \mathrm{a}$ & $2.423 \mathrm{a}$ & $658.11 \mathrm{~b}$ & $697.65 \mathrm{~b}$ & $176.23 \mathrm{~b}$ & $191.31 b$ \\
\hline \multicolumn{12}{|c|}{ Interaction (salinity $*$ seed priming) } \\
\hline \multirow{4}{*}{$0 \mathrm{mM}$} & $0.0 \mathrm{mM}$ & $9.06 \mathrm{~h}$ & $8.72 \mathrm{~h}$ & $31.230 \mathrm{i}$ & $29.980 \mathrm{i}$ & $2.230 \mathrm{f}$ & $2.097 \mathrm{f}$ & $600.91 \mathrm{fg}$ & $637.06 \mathrm{fg}$ & $144.52 \mathrm{ef}$ & $156.88 \mathrm{ef}$ \\
\hline & $0.1 \mathrm{mM}$ & $9.51 \mathrm{e}$ & $9.15 \mathrm{e}$ & 33.967 ef & 32.607 ef & $2.440 \mathrm{de}$ & $2.293 \mathrm{de}$ & $720.10 \mathrm{bc}$ & $763.42 \mathrm{bc}$ & $180.53 \mathrm{c}$ & $195.97 \mathrm{c}$ \\
\hline & $0.3 \mathrm{mM}$ & $10.05 \mathrm{a}$ & $9.68 \mathrm{a}$ & $35.800 \mathrm{~b}$ & & $7 \mathrm{de}$ & $2.320 \mathrm{cde}$ & $874.78 \mathrm{a}$ & $927.41 \mathrm{a}$ & $209.63 \mathrm{a}$ & $227.57 \mathrm{a}$ \\
\hline & $0.5 \mathrm{mM}$ & $9.74 \mathrm{~b}$ & $9.37 \mathrm{~b}$ & $34.300 \mathrm{de}$ & $32.927 \mathrm{de}$ & 2.507 bcde & $2.357 \mathrm{bcde}$ & $720.21 \mathrm{bc}$ & $763.54 \mathrm{bc}$ & $181.04 \mathrm{c}$ & $196.53 \mathrm{c}$ \\
\hline \multirow{4}{*}{$30 \mathrm{mM}$} & $0.0 \mathrm{mM}$ & $9.070 \mathrm{gh}$ & $8.73 \mathrm{gh}$ & $31.880 \mathrm{~h}$ & $30.603 \mathrm{~h}$ & $2.250 \mathrm{f}$ & $2.113 \mathrm{f}$ & 630.80 ef & 668.72 ef & $152.82 \mathrm{ef}$ & $165.89 \mathrm{ef}$ \\
\hline & $0.1 \mathrm{mM}$ & $9.52 \mathrm{e}$ & $9.16 \mathrm{e}$ & $33.617 \mathrm{fg}$ & $\mathrm{fg}$ & cde & cde & & $776.11 \mathrm{bc}$ & 185.4 & $5 \mathrm{bc}$ \\
\hline & $0.3 \mathrm{mM}$ & $10.07 \mathrm{a}$ & $9.69 \mathrm{a}$ & $37.130 \mathrm{a}$ & $35.643 \mathrm{a}$ & $2.600 \mathrm{abcd}$ & 2.443abcd & $843.85 \mathrm{a}$ & $894.57 \mathrm{a}$ & $212.83 \mathrm{a}$ & $231.03 \mathrm{a}$ \\
\hline & $0.5 \mathrm{mM}$ & $9.70 \mathrm{c}$ & $9.34 \mathrm{c}$ & $35.200 \mathrm{c}$ & $33.790 \mathrm{c}$ & $2.657 \mathrm{ab}$ & $2.497 \mathrm{ab}$ & $758.07 \mathrm{~b}$ & $803.63 \mathrm{~b}$ & $202.66 \mathrm{ab}$ & $220.00 \mathrm{ab}$ \\
\hline \multirow{4}{*}{$60 \mathrm{mM}$} & $0.0 \mathrm{mM}$ & $8.60 \mathrm{k}$ & $8.28 \mathrm{k}$ & $31.200 \mathrm{i}$ & $29.953 \mathrm{i}$ & 2.347 ef & $2.207 \mathrm{ef}$ & 511.19 hi & 541.89 hi & $136.24 \mathrm{fg}$ & $147.89 \mathrm{fg}$ \\
\hline & $0.1 \mathrm{mM}$ & $9.03 \mathrm{i}$ & $8.69 \mathrm{i}$ & $33.070 \mathrm{~g}$ & $31.747 \mathrm{~g}$ & 2.537abcd & 2.387abcd & $696.75 \mathrm{bcd}$ & $738.59 \mathrm{bcd}$ & $191.13 \mathrm{bc}$ & $207.47 \mathrm{bc}$ \\
\hline & $0.3 \mathrm{mM}$ & $9.54 \mathrm{~d}$ & $9.19 \mathrm{~d}$ & $35.930 \mathrm{~b}$ & $34.493 \mathrm{~b}$ & $2.677 \mathrm{a}$ & $2.517 \mathrm{a}$ & 672.91cde & $713.32 \mathrm{cde}$ & $184.26 b$ & $200.02 \mathrm{bc}$ \\
\hline & $0.5 \mathrm{mM}$ & $9.24 \mathrm{f}$ & $8.90 \mathrm{f}$ & $34.257 \mathrm{de}$ & $32.887 \mathrm{de}$ & $2.587 \mathrm{abcd}$ & $2.430 \mathrm{abcd}$ & $642.88 \mathrm{def}$ & $681.49 \mathrm{def}$ & $175.71 \mathrm{~cd}$ & $190.74 \mathrm{~cd}$ \\
\hline \multirow{4}{*}{$90 \mathrm{mM}$} & $0.0 \mathrm{mM}$ & 8.181 & 7.881 & $27.143 \mathrm{k}$ & $26.057 \mathrm{k}$ & $2.017 \mathrm{~g}$ & $1.897 \mathrm{~g}$ & $369.02 \mathrm{j}$ & $391.15 \mathrm{j}$ & $88.69 \mathrm{~h}$ & $96.27 \mathrm{~h}$ \\
\hline & $0.1 \mathrm{mM}$ & $8.59 \mathrm{k}$ & $8.27 \mathrm{k}$ & $29.633 \mathrm{j}$ & $28.450 \mathrm{j}$ & $2.250 \mathrm{f}$ & $2.113 \mathrm{f}$ & $466.56 \mathrm{i}$ & $494.54 \mathrm{i}$ & $119.22 \mathrm{~g}$ & $129.42 \mathrm{~g}$ \\
\hline & $0.3 \mathrm{mM}$ & $9.08 \mathrm{~g}$ & $8.74 \mathrm{~g}$ & $34.733 \mathrm{~cd}$ & $33.343 \mathrm{~cd}$ & $2.633 a b c$ & $2.473 \mathrm{abc}$ & $562.56 \mathrm{gh}$ & $596.29 \mathrm{gh}$ & $159.22 \mathrm{de}$ & $172.84 \mathrm{de}$ \\
\hline & $0.5 \mathrm{mM}$ & $8.79 \mathrm{j}$ & $8.47 \mathrm{j}$ & $31.300 \mathrm{i}$ & $30.047 \mathrm{i}$ & $2.563 \mathrm{abcd}$ & $2.410 \mathrm{abcd}$ & $511.28 \mathrm{hi}$ & 541.94 hi & 145.52 ef & 157.97 ef \\
\hline
\end{tabular}

Data of the trays experiment are demonstrated in table 3; the harmful effect of salinity on emergence percentage is evident. It decreased gradually with the augment in salinity level. Despite the salinity level, ASA seed priming contributed positively to emergence percentage. Concerning interaction between two factors, the lowest values recorded were for water primed seeds sown under high salinity conditions. Priming in $0.3 \mathrm{mM}$ ASA was the best treatment, it enhanced emergence under all salinity levels. It increased emergence by $34 \%$ and 33\% under control and high salinity conditions respectively, these findings agree with (Khan et al., 2009). Data for emergence percentage were employed in development of regression relationship to figure out the best between 2 vigor indices (Fig: 1). As it can be noticed, vigor index 1 is much more reliable to predict emergence \%. This is may be due to the involvement of seedling length in calculation of vigor index 1 . Therefore, the longer the seedling the more capable to emerge out of planting media it is. Values for transplant height significantly decrease as a result of increase in salinity level. While plant height ranged between 15.37 and 15.83 $\mathrm{cm}$ under control conditions, it was reduced to 4.83 and $4.97 \mathrm{~cm}$ under high salinity for the two seasons respectively. On the other hand, ASA seed priming improved plant height, the highest values were obtained from seeds primed with $0.3 \mathrm{mM}$ ASA, contributed positively to mean transplant height; it was 11.79 and $12.14 \mathrm{~cm}$ for the first and second season in that order.
The same trend was found in transplant dry weight data. Concerning the interaction between seed priming and salinity level, it can be found that priming with $0.3 \mathrm{mM}$ ASA was the most efficient dose. It mitigated the harmful impact of salinity on both of the two parameters. These results are in agreement with Cicek and Cakirlar (2002) studies on maize seedlings and (Khan et al.,, 2009) on hot pepper. The inhibition of plant growth is caused by cellular response to decreased water availability and high osmotic stress of external salts furthermore later on by toxic effects of excessive salt accumulation within the plant cells (Munns et al., 1995). Proline accumulation is an adopted mechanism for plants to combat salinity. It is obvious form data in table 3 that proline accumulation increased with the higher salinities. On the other hand, ASA contributed to keep these values down. The highest values recorded for proline content were 7.113 and 6.987 $\mathrm{mg} \mathrm{g}^{-1}$ for control seeds irrigated with high salinity solution. The effect of salinity on nutrient uptake was negative. $\mathrm{N}, \mathrm{P}$ and $\mathrm{K}$ uptake was negatively responding to salinity. The uptake of the 3 nutrients decreased with the increase in the salinity levels. The lowest value for the nutrients uptake recorded when transplants were irrigated with high salinity solution. While all of ASA priming treatment promoted nutrients uptake, $0.3 \mathrm{mM}$ ASA treatment surpassed. It increased $\mathrm{N}$ uptake about 87\%, P $61 \%$ and $\mathrm{K} 15 \%$ over control. This was definitely translated in height increase and biomass accumulation in transplants. These results are in line with (Gammoudi et 


\section{J. Plant Production, Mansoura Univ., Vol. 7(12), December, 2016}

al., 2016; Sakr et al., 2007). Finally, the harmful effects of salinity can be alleviated by seed priming in acetyl salicylic acid. This process involves many changes that promote seed vigor during germination and emergence under salinity stress (Ibrahim, 2016). Salicylic acid and it derivatives are well know agents with their role in abiotic stress alleviation. The results obtained in the current study stated that seed priming with acetyl salicylic acid, as one of salicylic acid derivatives, not only capable of improving seed germination and seedling establishment of pepper under favorable conditions, but also alleviated the deleterious effects of salinity stress during these stages. Many studies had similar results on various crop, (Khan et al.,, 2009) on hot pepper, (Osman and Salim, 2016) on snap bean, (Tari et al., 2002) on tomato. These positive effects may be referred to induction of enzymatic antioxidation system (Azooz, 2009) or due to antioxidation role and proline accumulation (Tari et al., 2002; Tari et al., 2004) or it may be due to its role in prevention of the decrease in growth promoters (IAA and cytokinin) levels (Shakirova et al., 2003) this is beside it stated role in enhancement of the photosynthetic rate and also maintenance of the Membranes stability (El Tayeb, 2005).

Table 3. Effect of salinity, acetyl salicylic acid seed priming and their interaction on physicochemical parameters of transplants during two seasons 2014 and 2015.

\begin{tabular}{|c|c|c|c|c|c|c|c|c|c|c|c|c|c|c|}
\hline \multirow{2}{*}{ Treatments } & \multirow{2}{*}{\multicolumn{2}{|c|}{$\begin{array}{c}\text { Emergence } \\
\%\end{array}$}} & \multirow{2}{*}{\multicolumn{2}{|c|}{$\begin{array}{l}\text { Transplant } \\
\text { Height }(\mathrm{cm})\end{array}$}} & \multirow{2}{*}{\multicolumn{2}{|c|}{$\begin{array}{l}\text { Transplant Dry } \\
\text { Weight (g) }\end{array}$}} & \multicolumn{8}{|c|}{ Leaf chemical characteristics } \\
\hline & & & & & & & \multicolumn{2}{|c|}{ Proline $\left(\mathrm{mg} \mathrm{g}^{-1}\right)$} & \multicolumn{2}{|c|}{$\mathbf{N}(\%)$} & \multicolumn{2}{|c|}{$\mathbf{P}(\%)$} & \multicolumn{2}{|c|}{$\mathbf{K}(\%)$} \\
\hline \begin{tabular}{lc} 
Salinity & \multicolumn{1}{c}{ Seed } \\
level & priming
\end{tabular} & $\begin{array}{c}1^{\text {st }} \\
\text { season }\end{array}$ & $\begin{array}{c}2^{\text {nd }} \\
\text { season }\end{array}$ & $\begin{array}{c}1^{\text {st }} \\
\text { season }\end{array}$ & $\begin{array}{c}2^{\text {nd }} \\
\text { season }\end{array}$ & $\begin{array}{c}1^{\text {st }} \\
\text { season }\end{array}$ & $\begin{array}{c}2^{\text {nd }} \\
\text { season }\end{array}$ & $\begin{array}{c}1^{\text {st }} \\
\text { season }\end{array}$ & $\begin{array}{c}2^{\text {nd }} \\
\text { season }\end{array}$ & $\begin{array}{c}1^{\text {st }} \\
\text { season }\end{array}$ & $\begin{array}{c}2^{\text {nd }} \\
\text { season }\end{array}$ & $\begin{array}{c}1^{\text {st }} \\
\text { season }\end{array}$ & $\begin{array}{c}2^{\text {nd }} \\
\text { season }\end{array}$ & $\begin{array}{c}1^{\text {st }} \\
\text { season }\end{array}$ & $\begin{array}{c}2^{\text {nd }} \\
\text { season }\end{array}$ \\
\hline \multicolumn{15}{|c|}{ Salinity level $(\mathrm{mM} \mathrm{NaCl})$} \\
\hline $0 \mathrm{mM}$ & $75.45 \mathrm{a}$ & $78.47 \mathrm{a}$ & $15.37 \mathrm{a}$ & $15.83 \mathrm{a}$ & $0.244 \mathrm{c}$ & $0.300 \mathrm{c}$ & $1.997 \mathrm{~d}$ & $1.961 \mathrm{~d}$ & $3.511 \mathrm{a}$ & $3.602 \mathrm{a}$ & $0.702 \mathrm{a}$ & $0.719 \mathrm{a}$ & $1.822 \mathrm{a}$ & $2.000 \mathrm{a}$ \\
\hline $30 \mathrm{mM}$ & $76.21 \mathrm{a}$ & $79.25 \mathrm{a}$ & $13.40 \mathrm{~b}$ & $13.80 \mathrm{~b}$ & $0.262 \mathrm{a}$ & $0.322 \mathrm{a}$ & $2.622 \mathrm{c}$ & $2.574 \mathrm{c}$ & $3.152 \mathrm{~b}$ & $3.235 \mathrm{~b}$ & $0.630 \mathrm{~b}$ & $0.648 b$ & $1.578 \mathrm{~b}$ & $1.735 \mathrm{~b}$ \\
\hline $60 \mathrm{mM}$ & $67.15 \mathrm{~b}$ & $69.84 \mathrm{~b}$ & $9.80 \mathrm{c}$ & $10.10 \mathrm{c}$ & $0.250 \mathrm{~b}$ & $0.307 \mathrm{~b}$ & $3.867 \mathrm{~b}$ & $3.798 b$ & $2.900 \mathrm{c}$ & $2.975 \mathrm{c}$ & $0.551 \mathrm{c}$ & $0.568 \mathrm{c}$ & $1.394 \mathrm{c}$ & $1.533 \mathrm{c}$ \\
\hline $90 \mathrm{mM}$ & $51.31 \mathrm{c}$ & $53.36 \mathrm{c}$ & $4.83 \mathrm{~d}$ & $4.97 \mathrm{~d}$ & $0.236 \mathrm{~d}$ & $0.291 \mathrm{~d}$ & $5.210 \mathrm{a}$ & $5.115 \mathrm{a}$ & $2.580 \mathrm{~d}$ & $2.648 \mathrm{~d}$ & $0.493 \mathrm{~d}$ & $0.505 \mathrm{~d}$ & $1.243 \mathrm{~d}$ & $1.364 \mathrm{~d}$ \\
\hline \multicolumn{15}{|c|}{ Seed priming (mM Acetyl Salicylic Acid) } \\
\hline
\end{tabular}

$0.0 \mathrm{mM} 58.47$ d 60.81 d 10.00 d 10.30 d 0.209 d 0.257 d 4.743 a 4.658 a 1.894 d 1.947 d 0.439 d 0.450 d 1.407 d 1.546 d $0.1 \mathrm{mM} 68.92$ b 71.67 b 10.67 c 10.99 c 0.238 c 0.293 c 3.274 b 3.214 b 3.453 b 3.542 b 0.603 c 0.621 c 1.493 c 1.639 c $0.3 \mathrm{mM} 78.16$ a 81.29 a 11.79 a 12.14 a 0.292 a 0.359 a 3.078 c 3.022 c 3.547 a 3.638 a 0.703 a 0.723 a 1.618 a 1.778 a $0.5 \mathrm{mM} 64.57$ c 67.15 c 10.94 b 11.27 b 0.253 b 0.311 b 2.600 d 2.554 d 3.249 c 3.333 c 0.629 b 0.647 b 1.520 b 1.669 b Interaction (salinity * seed priming)

zero $\quad 65.33$ ef 67.95 ef 14.85 b 15.29 b 0.204 h 0.251 h 2.867 f 2.817 f 2.200 h 2.260 h 0.530 h 0.540 g 1.703 d 1.870 d zero $\quad 0.1 \mathrm{mM} 77.00$ b 80.08 b 15.43 a 15.90 a 0.231 fg 0.285 fg 1.930 i 1.897 i 3.947 a 4.050 a 0.690 c 0.707 c 1.786 c 1.960 c $0.3 \mathrm{mM} 87.33$ a 90.83 a 15.65 a 16.12 a 0.291 b 0.358 b 1.473 j 1.443 j 3.967 a 4.067 a 0.850 a 0.873 a 1.963 a 2.157 a $0.5 \mathrm{mM} 72.14 \mathrm{~cd} 75.02$ d 15.55 a 16.02 a 0.250 e 0.307 e 1.717 i 1.687 i 3.930 a 4.030 a 0.737 b 0.757 b 1.833 b 2.013 b zero $\quad 65.99$ ef 68.63 ef 12.25 d 12.62 d 0.222 g 0.273 g 3.750 d 3.680 de 2.050 i 2.110 i 0.463 j 0.477 i 1.476 g $1.623 \mathrm{~g}$

$30 \mathrm{mM} \quad 0.1 \mathrm{mM} 77.77$ b 80.88 b 13.37 c 13.77 c 0.251 de 0.309 de 2.553 g 2.507 g 3.787 b 3.883 b 0.657 e 0.676 d $1.550 \mathrm{f} 1.706 \mathrm{f}$ $0.3 \mathrm{mM} 88.21$ a 91.73 a 14.63 b 15.07 b 0.312 a 0.384 a 2.230 h2 2.190 h3 3.827 b3 3.924 b 0.727 b 0.747 b 1.690 d 1.857 d $0.5 \mathrm{mM} 72.86$ c 75.77 c 13.33 c 13.74 c $0.261 \mathrm{~cd} 0.321 \mathrm{~cd} 1.953$ i 1.920 i $2.943 \mathrm{f} 3.020 \mathrm{f} 0.673$ d 0.693 c 1.597 e 1.753 e zero $\quad 58.15$ g 60.47 g 8.96 g 9.23 g 0.210 h 0.258 h 5.243 b5.150 b 1.760 j 1.810 j 0.403 k 0.413 j 1.293 j $1.423 \mathrm{j}$ $60 \mathrm{mM} \quad 0.1 \mathrm{mM} 68.53 \mathrm{de} 71.27 \mathrm{de} 9.78 \mathrm{f} \quad 10.07 \mathrm{f} \quad 0.241$ ef $0.296 \mathrm{ef} 3.770 \mathrm{~d} 3.700 \mathrm{~d} 3.216 \mathrm{~d} 3.297 \mathrm{~d} 0.563 \mathrm{~g} 0.583 \mathrm{f} 1.393 \mathrm{~h} 1.530 \mathrm{~h}$ $0.3 \mathrm{mM} 77.73$ b 80.84 b 10.71 e 11.03 e 0.299 b 0.367 b 3.507 e 3.447 e 3.383 c 3.470 c 0.653 e 0.673 d 1.490 g $1.640 \mathrm{~g}$ $0.5 \mathrm{mM} \quad 64.20 \mathrm{f} 66.77 \mathrm{f} 9.76 \mathrm{f} 10.05 \mathrm{f} 0.251$ e 0.308 de $2.947 \mathrm{f} 2.897 \mathrm{f} 3.240 \mathrm{~cd} 3.323 \mathrm{~d} 0.583 \mathrm{f} 0.603$ e $1.400 \mathrm{~h} 1.540 \mathrm{~h}$

zero $\quad 44.43 \mathrm{i} 46.20 \mathrm{i} 3.94 \mathrm{j} \quad 4.06 \mathrm{j} 0.200 \mathrm{~h} 0.246 \mathrm{~h} 7.113$ a 6.987 a $1.567 \mathrm{k} 1.607 \mathrm{k} 0.36010 .370 \mathrm{k} 1.15311 .2671$

$90 \mathrm{mM} \quad 0.1 \mathrm{mM} 52.36 \mathrm{~h} 54.45 \mathrm{~h} \quad 4.10 \mathrm{j} \quad 4.22 \mathrm{j} \quad 0.230 \mathrm{fg} 0.283 \mathrm{fg} 4.843 \mathrm{c} 4.753$ c $2.860 \mathrm{~g} 2.937 \mathrm{~g} 0.503$ i $0.517 \mathrm{~h} 1.240 \mathrm{k} 1.360 \mathrm{k}$ $0.3 \mathrm{mM} 59.39 \mathrm{~g} 61.76 \mathrm{~g} \quad 6.15 \mathrm{~h} \quad 6.33 \mathrm{~h} 0.264$ c 0.325 c 5.100 b 5.007 b 3.010 e 3.090 e 0.583 f 0.600 e 1.330 i $1.460 \mathrm{i}$

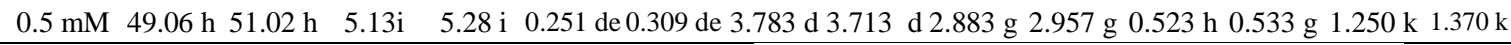
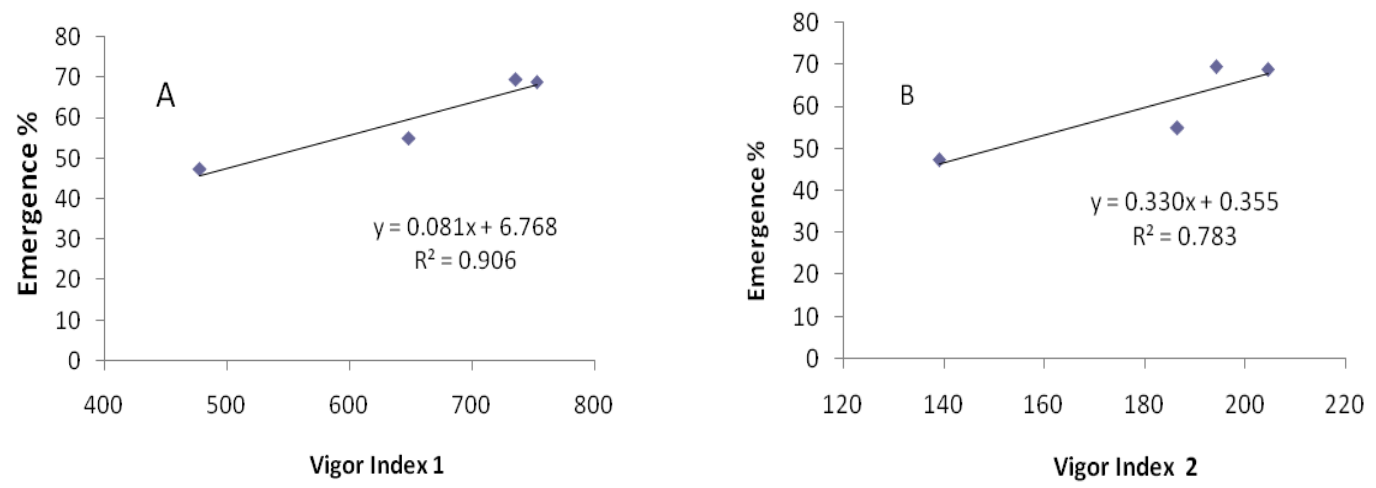

Fig. 1. regression relationship between seedling vigor indices and emergence percentage, average of two seasons 2014 and 2015 were employed. 


\section{REFERENCES}

A.O.A.C. (1992) Official Methods of Analysis Association of Official Analysis Chemists, $\left(12^{\text {th }}\right.$ ED.), Washington, DC. U.S.A.

Abdul-Baki A.A.and Anderson J.D. (1973) Vigour determination in soybean and seed multiple criteria. Crop science 13:630-633.

Aktas H., Abak K.and Cakmak I. (2006) Genotypic variation in the response of pepper to salinity. Sci. Hortic. 110:260-266.

Ashraf M.and Foolad M.R. (2005) Pre-Sowing Seed Treatment-A Shotgun Approach to Improve Germination, Plant Growth, and Crop Yield Under Saline and Non-Saline Conditions. Advances in Agronomy 88:223-271.

Azooz M.M. (2009) Salt stress mitigation by seed priming with salicylic acid in two faba bean genotypes differing in salt tolerance. Int. J. .Agric Biol. 11: 343-350.

Bates L.S., Waldern R.P.and Teare I.D. (1973) Rapid determination of free proline for water stress studies. Plant and Soil 39: 205-207.

Cantliffe D.J. (2003) Seed Enhancements. Acta Horticulturae 607:53-59.

Chapman H.D.and Pratt P.F. (1961) Methods of analysis for soils, plants and water University of California, Berkeley.

Chartzoulakis K.and Klapaki G. (2000) Response of two greenhouse pepper hybrids to $\mathrm{NaCl}$ salinity during different growth stages. Scientia Horticulturae 86:247-260.

Cicek N.and Cakirlar H. (2002) The effect of salinity on some physiological parameters in two maize cultivars. Bulgarian Journal of Plant Physiology 28:66-74.

Cornillon P.and Palloix A. (1995) Influence de la salinité et de la température du substrat sur la croissance et la nutrition du piment. Fruits 50:469-471.

Demir I.and Okcu G. (2004) Aerated hydration treatment for improved germination and seedling growth in aubergine (Solanum melongena) and pepper (Capsicum annum). Annals of Applied Biology 144:121-123.

Edwards R.L.and Sundstorm F.J. (1987) After ripening and harvesting effects on Tabasco pepper seed germination performance. HortScience 22:473475.

El Tayeb M.A. (2005) Response of barley grains to the interactive effect of salinity and salicylic acid. Plant Growth Regul. 45:215-224.

Ellis R.H.and Roberts E.H. (1981) The quantification of aging and survival in orthodox seeds. Seed Sci. Technol. 9: 373-409.

Farooq M., Basra S.M.A., Hafeez K.and Ahmad N. (2005) Thermal hardening: a new seed vigor enhancement tool in rice. Journal of Integrative Plant Biology 47:187-193.
Gain P., Mannon M.A., Pal P.S., Hossien M.M.and Parvi S. (2004) Effect of salinity on some yield attribution of rice. Pak J. Biol Sci. 7:760-762.

Gammoudi N., Yahia L.B., Lachiheb B.and Ferchichi A. (2016) Salt response in pepper (Capsicum annuum L.): Components of photosynthesis inhibition, proline accumulation, and $\mathrm{K}^{+} / \mathrm{Na}^{+}$ selectivity. Journal of Arid land Agriculture 2: 1-12.

Ibrahim E.A. (2016) Seed priming to alleviate salinity stress in germinating seeds. Journal of Plant Physiology 192:38-46.

Iroka C.F., Chukwuma M.O.and Izundu A.I. (2016) Evaluation of salt tolerance of some varieties of Capsicum species during germination using morphometric features International Journal of Botany Studies 1:29-33.

ISTA. (2011) International rules for seed testing, Zurich: Switzerland.

Khan H.A.K., M.A. Pervez, Ayub C.M., Ziaf K., Balal R.M., Shahid M.A.and Akhtar N. (2009) Hormonal priming alleviates salt stress in hot Pepper (Capsicum annuиm L.). Soil \& Environ. 28:130-135.

Korkmaz A. (2005) Inclusion of acetyl salicylic acid and methyl jasmonate into the priming solution improves low-temperature germination and emergence of sweet pepper. HortScience 40:197-200.

Korkmaz A., Uzunlu M.and Demirkiran A.R. (2007) Treatment with acetyl salicylic acid protects muskmelon seedlings against drought stress. Acta Physiologiae Plantarum 29:503-508.

Kurunc A., Unlukara A.and Cemek B. (2011) Salinity and drought affect yield response of bell pepper similarly. Acta Agric. Scand. Sect., B: Soil Plant Sci. 61:514-522

Lee S.S., Kim J.H., Hong S.B., Yuu S.H.and Park E.H. (1998) Priming effect of rice seeds on seedling establishment under adverse soil conditions. Korean Journal of Crop Science 43:194-198.

Maas E.V.and Hoffman G.J. (1977) Crop salt tolerance - current assessment. J. Irrig. Drain. Div. 103: 115-134.

Mohamed A.A., Eichler-Lobermann B.and Schnug E. (2007) Response of crops to salinity under Egyptian conditions: a review. Landbauforschung Volkenrode 57:119- 125.

Munns R., Schachtman D.and Condon A. (1995) The significance of a two-phase growth response to salinity in wheat and barley. Functional Plant Biology 22:561-569.

Munns R.and Tester M. (2008) Mechanisms of salinity tolerance. Annu. Rev. Plant Biol. 59:651-681.

Osman H.S.and Salim B.B.M. (2016) Influence of exogenous application of some phytoprotectants on growth, yield and pod quality of snap bean under $\mathrm{NaCl}$ salinity. Annals of Agricultural Science 61: 1-13. 


\section{J. Plant Production, Mansoura Univ., Vol. 7(12), December, 2016}

Pill W.G.and Fieldhouse D.G. (1982) Emergence of pregerminated tomato seed stored in gels up to 20 days at low temperatures. journal of American Society of Horticultural Science 107:722-725.

Pinero M.C., Houdusse F., Garcia-Mina J.M., Garnica M.and del Amor F.M. (2014) Regulation of hormonal responses of sweet pepper as affected by salinity and elevated $\mathrm{CO}_{2}$ concentration. Physiol. Plant. 151 375-389.

Rhoades J.D., Kandiah A.and Mashali A.M. (1992) The use of saline waters for crop production FAO Rome.

Sakr M., El-Emery M., Fouda R.and Mowafy M. (2007) Role of some antioxidants in alleviating soil salinity stress. J Agric Sci Mansoura Univ 32:9751-9763.

Senaratna T., Touchell D., Bunn E.and Dixon K. (2000) Acetyl salicylic acid (Aspirin) and salicylic acid induce multiple stress tolerance in bean and tomato plants. Plant Growth Regulation 30:157-161.
Shakirova F.M., Sakhabutdinova A.R., Bezrukova M.V., Fatkhutdinova R.A.and Fatkhutdinova D.R. (2003) Changes in the hormonal status of wheat seedlings induced by salicylic acid and salinity. Plant Sci. 164:317-322.

Shakirova F.M.and Sakhabutdinova D.R. (2003) Changes in the hormonal status of wheat seedlings induced by salicylic acid and salinity. Plant Sci. 164:317-322.

Tari I., Csiszar J., Szalai G., Horvath F., Pecsvaradi A., Kiss G., Szepesi A., Szabo M.and Erdei L. (2002) Acclimation of tomato plants to salinity stress after a salicylic acid pre-treatment. Acta. Biol. Szegediensis 46:55-56.

Tari I., Csiszar J., Szalai G., Horvath F., Pecsvaradi A., Kiss G., Szepesi A., Szabo M.and Erdei L. (2004) Influence of salicylic acid on salt stress acclimation of tomato plants: oxidative stress responses and osmotic adaptation. Acta Physiol. Plant 26S:237.

Yildirim E.and Güvenç İ. (2006) Salt tolerance of pepper cultivars during germination and seedling growth. Turkish journal of agriculture and forestry 30:347-353.

\section{الحد من الأثار الضـارة للملوحة على انبـات و نمو شتلات القلفل الحلو عن طريق التهيئة في محلول الاستيل

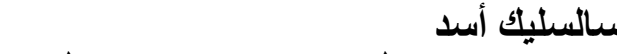

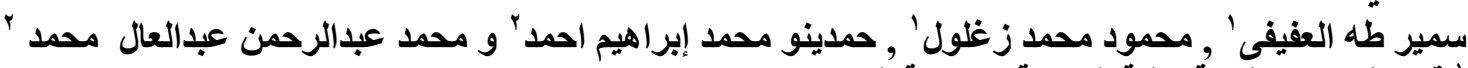

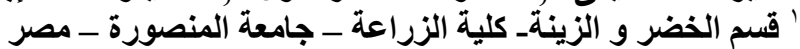

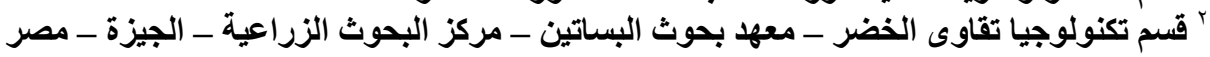

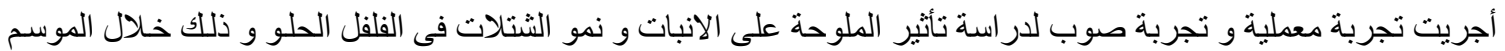

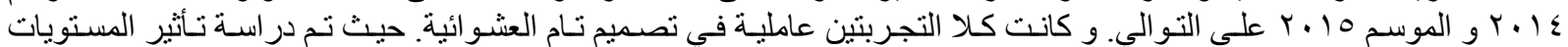

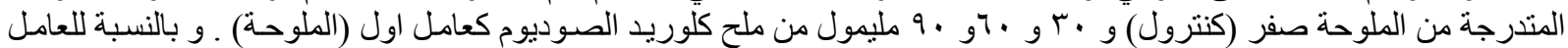

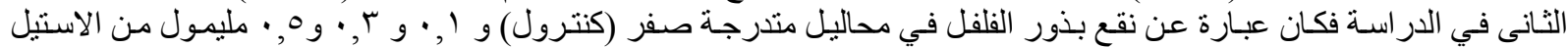

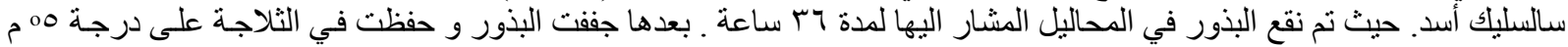

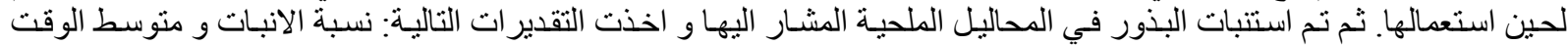

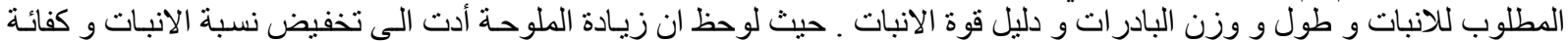

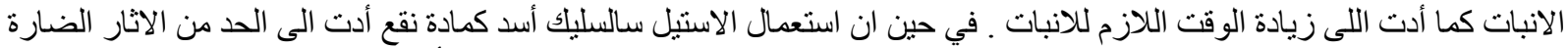

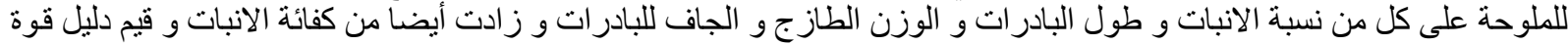

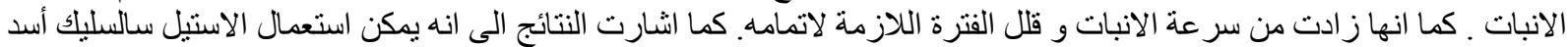

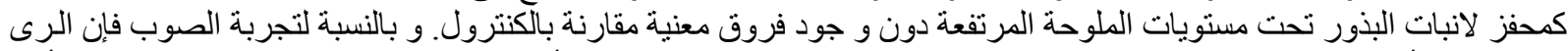

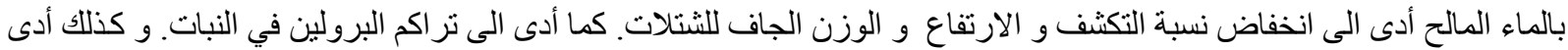

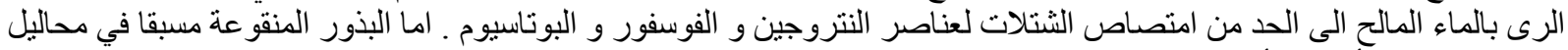

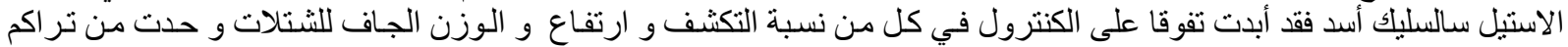

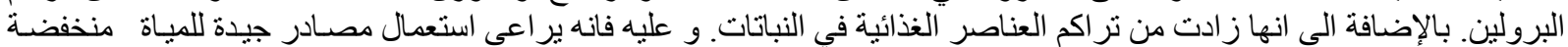

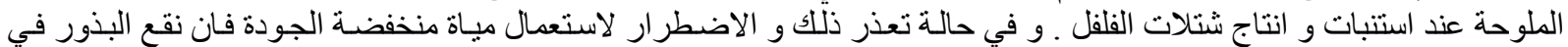
محلول الاستيل سالسليك أسد (يفضل ؟اج, • مليمول ) لمدة بس ساعة يعد اختيار ا مناسبا لتحسين الانبات و جودة الثتلات الناتجة. 